\title{
Usefulness of Color Coding Resected Samples from a Pancreaticoduodenectomy with Tissue Marking Dyes for a Detailed Examination of Surgical Margin Surrounding the Uncinate Process of the Pancreas
}

\author{
Satoshi Mizutani ${ }^{1}$, Hideyuki Suzuki ${ }^{1}$, Takayuki Aimoto', Seiji Yamagishi ${ }^{1}$, \\ Keisuke Mishima ${ }^{1}$, Masanori Watanabe ${ }^{1}$, Yasuhiko Kitayama ${ }^{2}$, Norio Motoda ${ }^{2}$, \\ Saiko Isshiki ${ }^{3}$ and Eiji Uchida ${ }^{4}$

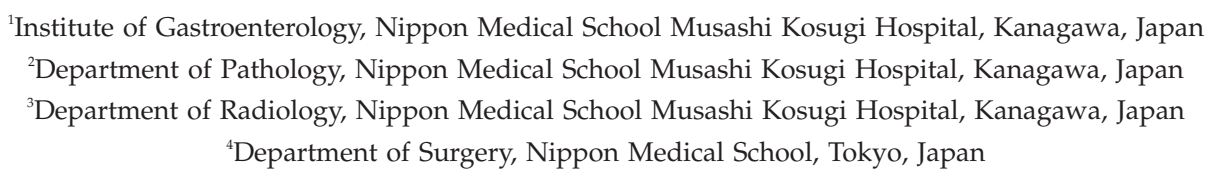

Background: Characteristics of a cancer-positive margin around a resected uncinate process of the pancreas (MUP) due to a pancreticoduodenectomy are difficult to understand by standardized evaluation because of its complex anatomy.

The purposes of this study were to subclassify the MUP with tissue marking dyes of different colors and to identify the characteristics of sites that showed positivity for cancer cells in patients with pancreatic head carcinoma who underwent circumferential superior mesenteric arterial nerve plexuspreserving pancreaticoduodenectomy. Results of this evaluation were used to review operation procedures and perioperative methods.

Method: We divided the MUP into 4 sections and stained each section with a different color. These sections were the pancreatic head nerve plexus margin (Area A), portal vein groove margin (Area B), superior mesenteric artery margin (Area $C$ ), and left of the superior mesenteric artery margin (Area D). The subjects evaluated were 45 patients who had carcinoma of the pancreatic head and were treated with circumferential superior mesenteric arterial nerve plexus-preserving pancreaticoduodenectomy.

Results: Of the 45 patients, nine cases (90\%) of incomplete resection showed cancer-positivity in the MUP. Among the 4 sections of the MUP, the most cases of positive results [MUP(+)] were found in Area B, with Area $\mathrm{A}(+), 0$ case; Area $\mathrm{B}(+), 6$ cases; Area $\mathrm{C}(+), 2$ cases; and Area $\mathrm{D}(+), 3$ cases (total, 11 sites in 9 patients). Relapse occurred in 7 of the 9 patients with MUP(+). Local recurrence was observed as initial relapse in all 3 patients with Area $\mathrm{D}(+)$. In contrast, the most common site of recurrence other than that in patients with Area $\mathrm{D}(+)$ was the liver.

Conclusion: By subclassifying the MUP with tissue marking dyes of different colors, we could confirm regional characteristics of $\mathrm{MUP}(+)$. As a result, circumferential superior mesenteric arterial nerve plexuspreserving pancreticoduodenectomy was able to be performed in R0 operations in selected patients while a better postoperative quality of life was maintained. Furthermore, Area $D(+)$ represents an extension beyond the limit of the local disease and may indicate the need for early aggressive adjuvant chemotherapy. (J Nippon Med Sch 2017; 84: 32-40)

Key words: pancreatic head carcinoma, pancreaticoduodenectomy, mesopancreas, tissue marking dye, surgical margin

Correspondence to Satoshi Mizutani, MD, Institute of Gastroenterology, Nippon Medical School Musashi Kosugi Hospital, 1-396 Kosugi-cho, Nakahara-ku, Kawasaki, Kanagawa 211-8533, Japan

E-mail: mizutani@nms.ac.jp

Journal Website (http://www.nms.ac.jp/jnms/) 

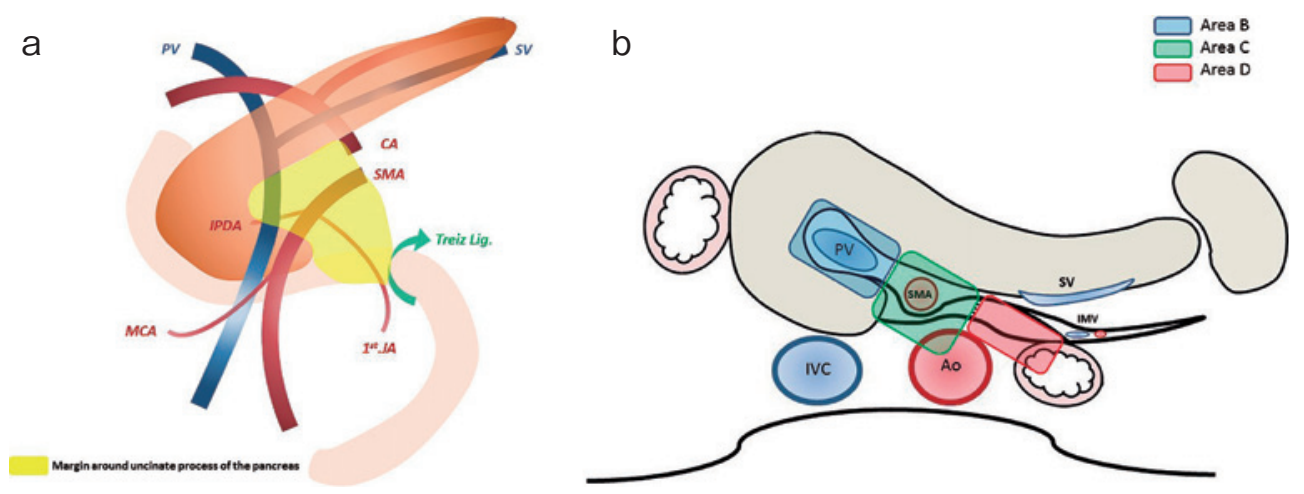

Fig. 1 a: The margin of the uncinate process of the pancreas (MUP) (yellow area) is shown in the diagram. The caudal margin of MUP is determined by the horizontal portion of duodenum.

b: Subclassification of the MUP is shown in a cross-sectional image of the abdomen. Three quadrangular regions stained with different colors represent each section of the MUP.

\section{Introduction}

Pancreaticoduodenectomy (PD) is the standard surgery for carcinoma of the pancreatic head. For patients with this disease, the best hope for a cure is complete histopathological resection ${ }^{1-3}$. To determine the success of complete histopathological resection, the most important factor is a resected peripancreatic tissue margin that tests negative for the involvement of cancer cells. Hence, improving the operative procedures in PD to ensure complete excision, after which histopathological examination shows no residual tumor (R0), is important $t^{5-7}$.

Surgical margins resected with PD consist of the pancreatic transection margin, bile duct cut margin, enteric margin, anterior surface, posterior margin, hepatoduodenal ligament margin, and the margin around the uncinate process of the pancreas (abbreviated hereafter as "MUP"). In these margins, characteristics of a cut end in which histopathological examination has shown cancer cells to be involved with the MUP [MUP(+)] are difficult to understand through standardized evaluation, because the MUP is a wide, complicated, and anatomically and embryologically 3-dimensional structure intricately passed through by the superior mesenteric artery (SMA), superior mesenteric vein (SMV), and many nerve plexuses around the pancreatic head (Fig. 1a) ${ }^{4-8}$.

Therefore, we devised a subclassification of the MUP and performed a histopathological review of the characteristics of cancer-positive sites in $\mathrm{MUP}(+)$ cases. The MUP was divided into 4 sections, each of which was stained with a tissue marking dye (TMD) of a different color (Fig. 1b). Staining different parts of the MUP with different colors of TMD allowed for subclassification of the MUP and confirmation of regional characteristics of cancer-positive MUP. Subclassifying the MUP was most difficult along the SMV from the area along the SMA. In most cases, the border between the SMV area and SMA area in the resected sample is not easily identified. Determining whether the stump of the MUP was the true surface or a desquamated surface in the prepared slides was difficult. Furthermore, the significance of positivity for cancer in the MUP differed greatly, depending on where the cancer cells were observed on the MUP site. For example, positivity for cancer observed along the boundary between the SMV or SMA adventitia (marginal) was more clinically significant than was positivity observed along the boundary between consecutive fat tissues and lymph nodes ${ }^{9,10}$.

In 2008, circumferential SMA nerve plexus (PLsma)preserving $\mathrm{PD}$ was introduced for R0 operations at our institution, in an effort to maintain patients' postoperative quality of life. Indications for PLsma-preserving PD were determined on the basis of the previously reported enhanced computed tomographic (CT) criteria for PLsma invasion $^{11-13}$. Circumferential PLsma-preserving PD was performed for all patients who were confirmed as not having obvious PLsma invasion. However, circumferential PLsma-preserving PD is also associated with a high risk of cancer positivity in the dissected peripancreatic tissue margin. This method of surgery also highlights the difficulty of an unclear MUP at the border between the PLsma and the second section of the pancreatic head nerve plexus in the resected samples ${ }^{14-16}$.

The purposes of this study were to subclassify the MUP with TMDs of different colors and to identify the 


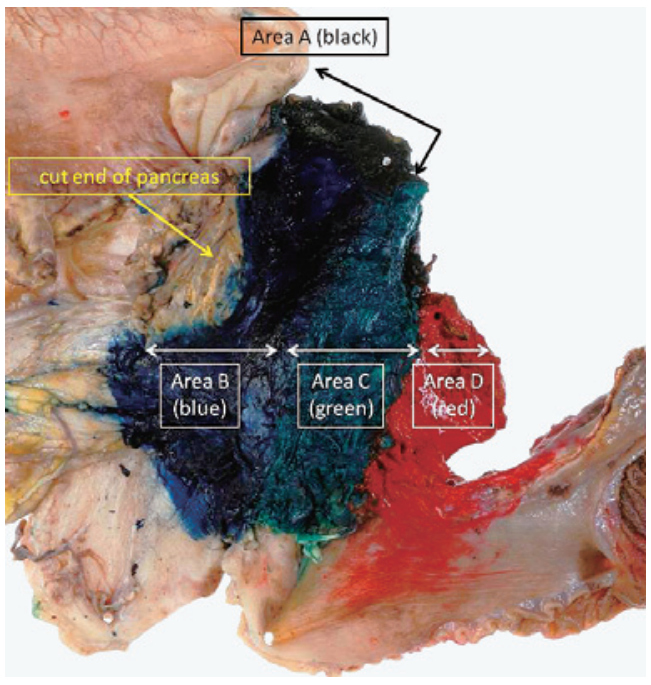

Fig. 2 Each MUP section is highlighted by TMD staining after formalin fixation. Area D (red) is markedly smaller after formalin treatment.

characteristics of sites identified as $\mathrm{MUP}(+)$ in patients with carcinoma of the pancreatic head who received circumferential PLsma-preserving PD. Results of this evaluation were also used to review operative procedures and perioperative methods.

\section{Method}

Anatomical Classification of the MUP According to Japanese General Rules for the Study of Pancreatic Cancer and the National Comprehensive Cancer Network Guidelines

According to the Japanese General Rules for the Study of Pancreatic Cancer ${ }^{17}$, the resected margin in pancreatic carcinoma is classified into 3 sections: the pancreatic cut end margin, the bile duct cut end margin, and the dissected peripancreatic tissue margin. The MUP defined in this study corresponds approximately to the remaining area after the anterior surface, posterior margin, and hepatoduodenal ligament margin are removed from the dissected peripancreatic tissue margin.

In contrast, the resection margin in pancreatic carcinoma according to the National Comprehensive Cancer Network (NCCN) practice guidelines on oncology for pancreatic adenocarcinoma ${ }^{18}$ is composed of the pancreatic transection margin, bile duct margin, enteric margin, anterior surface, posterior margin, SMA margin, and portal vein (PV) groove margin. The MUP corresponds to the total extent of the PV groove margin, SMA margin, and the area left of the SMA margin to the ligament of Treitz (Fig. 1a, b).

\section{Subclassification of the MUP}

We divided the MUP into the following 4 subsections on the basis of the NCCN guidelines and various references $^{6-9,18-20}$ (Fig. 1b, 2), namely (1) the area around the first portion of the pancreatic head nerve plexus, Area A (black); (2) the area around the PV groove margin, Area B (blue); (3) the area around the SMA margin, Area C (green); and (4) the area left of the SMA margin to the ligament of Treitz, Area D (red): Each of these sections of the MUP in the resected samples were stained with TMDs of different color, and, as a result, a detailed histopathological evaluation of the cut margin was able to be performed (Fig. 2).

Surgical Technique and Histopathological Preparation In combination with the circumferential PLsmapreserving method, we introduced a left posterior approach for a PD with total excision of the mesopancreas and circumferential lymphadenectomy around the SMA while preserving the circumferential PLsma in cases of carcinoma of the pancreatic head ${ }^{21-23}$. The second portion of the pancreatic head nerve plexus (PLPhII) was determined to be the left margin in resected samples in conventional PD. However, this tissue (fat tissue, lymph vessels, lymph nodes, and nerves) extends beyond the SMA margin (Area C) to the ligament of Treitz, and this section (Area D) was referred to as the "mesopancreas" together with the SMA margin (Area C) ${ }^{5-8,19,20,24}$ (Fig. 1-5). Thus, the entire Area D can be dissected in the left posterior approach for PD.

In the PLsma-preserving PD, the surface of Area $C$ is the border between the PLsma and the PLPhII (Fig. 4, 5). As TMDs for samples resected with PD, dyes of the Davidson Marking System (Bradley Products, Inc., Bloomington, MN, USA) were used. To prevent misidentification of each edge of the MUP, border points were marked with surgical clips during the operation. In particular, for the border between Area B and Area C, the marking clips were attached when the SMA was lifted and isolated by vessel taping. So that the resected samples could be satisfactorily stained, the TMDs were applied under both raw and formalin-fixed conditions.

Data from 45 patients, who had carcinoma of the pancreatic head without obvious PLsma invasion and had been treated with the left posterior approach for PD while preserving the circumferential PLsma, were evaluated from 2008 through 2014, with a focus on TMD staining. Indications for circumferential PLsma-preserving PD were determined on the basis of enhanced CT criteria for PLsma invasion as previously reported ${ }^{11-13}$. 

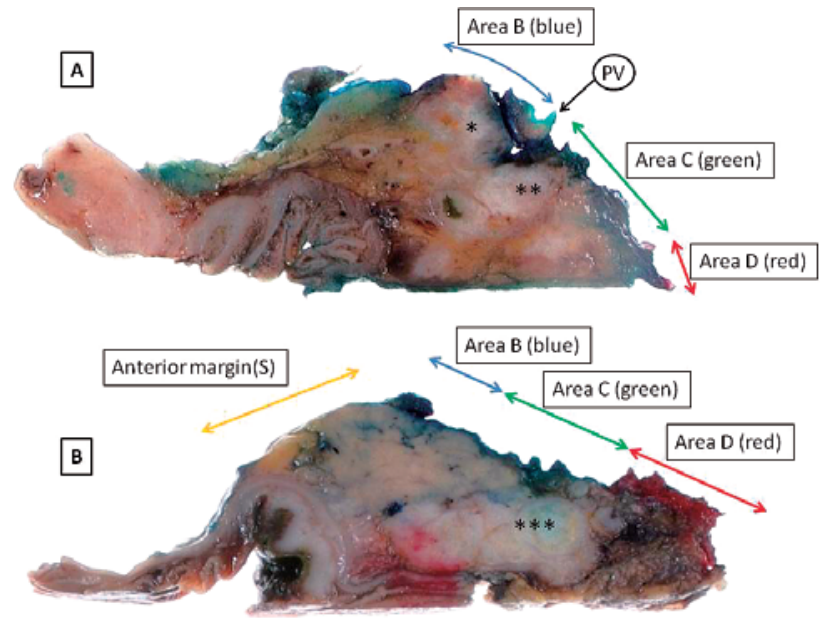

Fig. 3 Sections are detached parallel to the axial lines. A: Cancer cells (white mass) invading fat tissue surrounding the SMV contiguous with the anterior pancreatic capsule (Area B) $\left(^{*}\right)$ and PLPhII (Area C) $\left(^{* *}\right)$.

B: A greater amount of mesopancreas to the left of PhPLII (Area D) could not be dissected without LPAPD. Cancer cells invading the PLPhII (Area C) $\left.{ }^{(* *}\right)$.

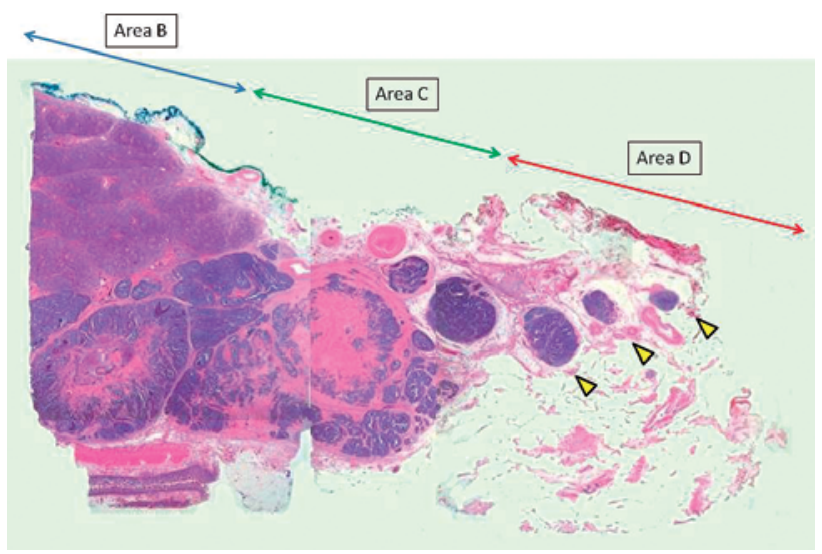

Fig. 4 Metastatic lymph nodes are observed in Area D. The patient was diagnosed with pancreatic neuroendocrine carcinoma (NETG3) and underwent surgery. (loupe image)

\section{Results}

On the basis of the Japanese General Rules for the Study of Pancreatic Cancer ${ }^{17}$, cases of pancreatic cancer were divided into stages in 45 patients as follows: stage II, 3 cases; stage III, 11 cases; stage IVa, 23 cases; and stage $\mathrm{IVb}, 8$ cases. The PV or SMV resection and reconstruction was performed for 15 patients (33.3\%). Histopathologic examination revealed serosal invasion in 30 cases, retroperitoneal invasion in 24 cases, duodenal invasion in 20 cases, common bile duct invasion in 19 cases, PV invasion in 8 cases, major arterial invasion in 1 case (accessory right hepatic artery), extra pancreatic nerve plexus

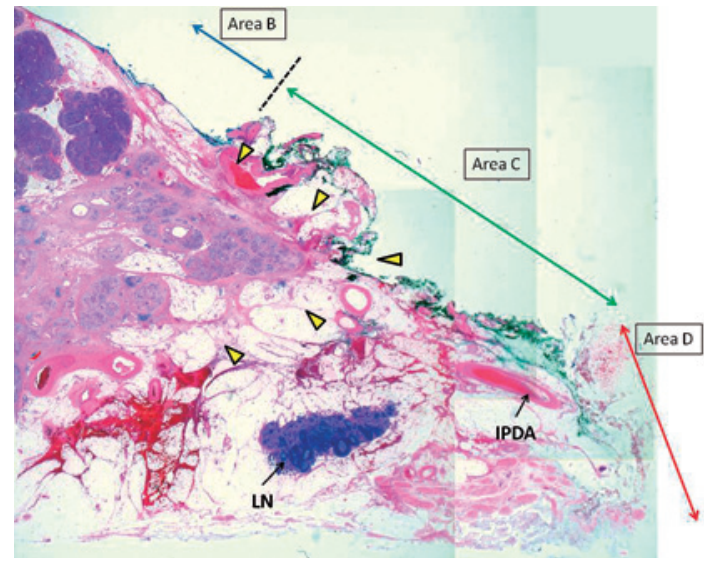

Fig. 5 Cancer cells invading the PLPhII (Area C: yellow arrows), but were not exposed on the surface at the border between PLsma and PLPhII [Area C $(-)$ ]. (loupe image)

Table 1 Histopathological examination of excised specimen

\begin{tabular}{ll}
\hline R0 & $35 / 45(77.8 \%)$ \\
R1 & $10 / 45$ \\
\hline MUP (+) & $9 / 45$ \\
MUP (-) & $2 / 45$ \\
\hline Area A (+) & $0 / 9$ \\
Area B (+) & $6 / 9$ \\
Area C (+) & $2 / 9$ \\
Area D (+) & $3 / 9$ \\
\hline MUP indicates margin around \\
the uncinated process of the \\
pancreas.
\end{tabular}

invasion in 19 cases (42.2\%), and other organ invasion in 0 cases. The rate of PL invasion was $42.2 \%$. Patients were excluded from this study if PLsma invasion had been preoperatively diagnosed. The PLPhII accounted for most cases of extra pancreatic nerve plexus invasion (14 of 19; $73.7 \%$ ). No patient had profuse or severe diarrhea after surgery, and all but 5 patients received adjuvant chemotherapy. The histopathological R0 rate was $77.8 \%$ (35 patients).

Of 10 cases of incomplete resection (R1), 9 (90\%) showed cancer positivity in the MUP (Table 1). When the $\mathrm{MUP}(+)$ cases were subclassified, only 2 were in Area C (4.4\%). Among the 4 MUP sections, the one with the most $\mathrm{MUP}(+)$ sites (total, 11 sites in 9 patients) was Area B (6 sites, Fig. 6), which was followed by Area D $(+)$ (3 sites) and Area $\mathrm{C}(+)$ (2 sites) (Fig. 7); (Table 1). Two pa- 


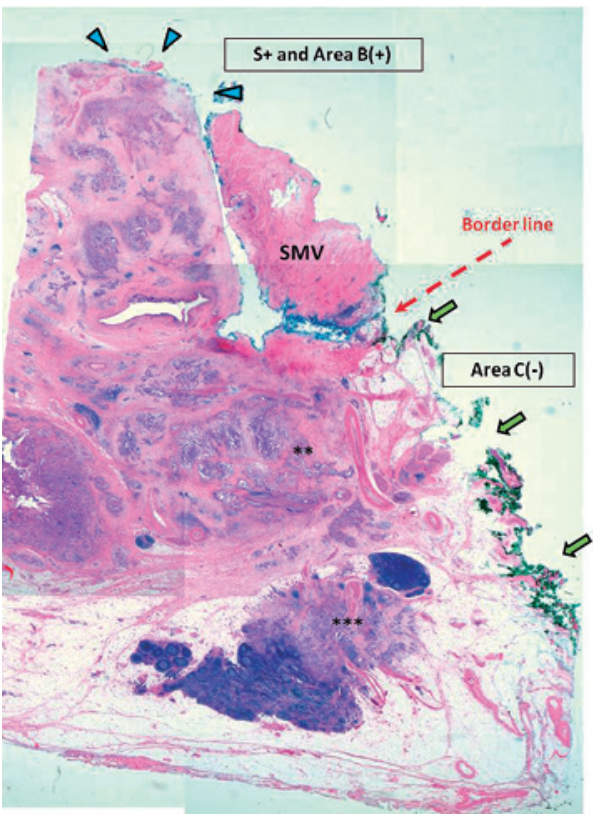

Fig. 6 Exposed cancer cells in fat tissue surrounding the SMV (blue arrows) [S+ and Area B (+)], and invading PLPhII (Area C) $\left.{ }^{* *}\right)$. Furthermore, a metastatic lymph node $\left.{ }^{* * *}\right)$ is seen rupturing tissue in PLPhII (Area C). Area C margin is negative (green arrow) (loupe image).

tients had multiple MUP(+) sites (1 patient with Areas B and $C$ and 1 patient with Areas $C$ and D) (Table 1). In Area $\mathrm{B}(+)$ cases, cancer cells were visible on the surface of the fat tissue resected around the SMV and PV, beyond the exposed serosa, and contiguous with invasion of the root of the mesentery of the transverse colon (Fig. 6).

The median follow-up period was 502 days, and disease relapsed in 25 patients $(55.6 \%)$. The primary type and location of recurrence after surgery in most patients (18 of 25 patients) was metastasis in the liver. Disease relapsed in 7 of 9 patients with $\mathrm{MUP}(+)$ cases $(77.8 \%)$. The site of initial recurrence differed significantly between patients with R0 and patients with R1 (Table 2); in patients with $\mathrm{R} 0$ the initial recurrence was never local recurrence. However, initial recurrence was localized in all patients with Area $\mathrm{D}(+)$ cases $(\mathrm{n}=3)$ but was not localized in most other patients with MUP(+) cases (Table 2).

\section{Discussion}

Surgical margins resected with PD consisted of the pancreatic transection margin, bile duct cut margin, enteric margin, anterior surface, posterior margin, hepatoduodenal ligament margin, and the MUP ${ }^{17,18,25}$. In these margins, determining exposed cancer cells was most difficult in

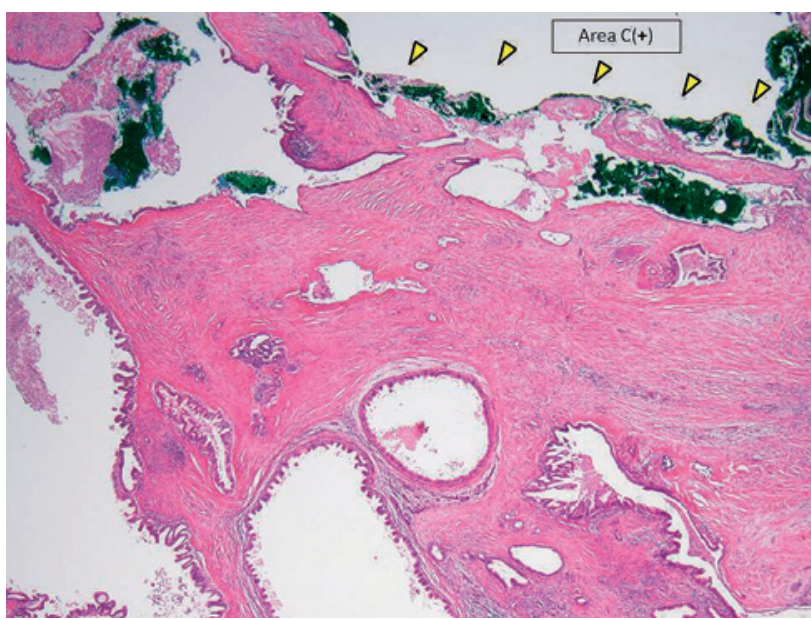

Fig. 7 Glandular tumors on the green line in PLPhII [Area C (+)]. (×2 magnification)

the MUP in histological preparations, because of the poorly defined edge of the margin and anatomic complexity ${ }^{5,6,8-10}$. By clarifying the characteristics of MUP(+) and determining the causes of positivity in the margin of specific sites, inadequacies in surgical procedures and limitations in local treatment can be differentiated. However, distinguishing whether the stump of the MUP is the true surface or a desquamated surface in prepared slides is difficult. Because these difficulties make the adequacy of operative procedures more difficult to determine, surgical techniques should be improved for $\mathrm{MUP}(+)$ cases, which is the most important factor in preventing $\mathrm{R} 0 \mathrm{op}-$ eration. Therefore, we subclassified the MUP and examined the characteristics of cancer-positive sites in MUP(+) cases.

Although some guidelines regarding the surgical margin have been issued in western countries ${ }^{18,25}$, subclassification is currently based on the NCCN guidelines ${ }^{18}$. The MUP corresponding to the Japanese General Rules for the Study of Pancreatic Cancer and the NCCN guidelines is described in the Methods section of the present article. The MUP subclassification standards were set according to the definition of margins in the NCCN guidelines ${ }^{18}$ because they closely coincide with the "mesopancreas" anatomically ${ }^{5-8,19}$. This MUP subclassification was able to be sufficiently confirmed because localized diagnoses of the MUP were possible and the presence of a positive margin was able to be clearly determined.

The first anatomical concept of the mesopancreas was published by Gockel et al. in $2007^{\circ}$. Popescu and Dumitrascu suggested that the mesopancreas is an anatomical space with the following limits: anterior, the posterior 
Table 2 Examination of recurrence of post-operative patients

\begin{tabular}{|c|c|c|c|c|}
\hline \multicolumn{5}{|l|}{ Recurrence } \\
\hline All & \multicolumn{4}{|c|}{$25 / 45(55.6 \%)$} \\
\hline R0 (35) & \multicolumn{4}{|c|}{$17 / 45$} \\
\hline R1 (10) & \multicolumn{4}{|c|}{$8 / 10$} \\
\hline R1 [MUP (+)] & \multicolumn{4}{|c|}{$7 / 9$} \\
\hline \multicolumn{5}{|l|}{ Initial recurrent site } \\
\hline Local & \multicolumn{4}{|c|}{$3 / 25$} \\
\hline Liver & \multicolumn{4}{|c|}{$18 / 25$} \\
\hline Lung & \multicolumn{4}{|c|}{$5 / 25$} \\
\hline Lymph node & \multicolumn{4}{|c|}{$4 / 25$} \\
\hline Initial recurrent site & \multicolumn{3}{|c|}{ R0 } & R1 \\
\hline Local & \multicolumn{3}{|c|}{0} & 3 \\
\hline Liver & \multicolumn{3}{|c|}{15} & 3 \\
\hline Lung & \multicolumn{3}{|c|}{4} & 1 \\
\hline Lymph node & \multicolumn{3}{|c|}{2} & 2 \\
\hline \multicolumn{5}{|l|}{ Recurrence based on MUP (+) site } \\
\hline Recurrence in Area B (+) & \multicolumn{4}{|c|}{$3 / 6$} \\
\hline Recurrence in Area C (+) & \multicolumn{4}{|c|}{$1 / 2$} \\
\hline Recurrence in Area D (+) & \multicolumn{4}{|c|}{$3 / 3$} \\
\hline Initial recurrent site based on MUP (+) site & Lung & Liver & Local & Lymph node \\
\hline Recurrence in Area B (+) $(n=3)$ & 1 & 2 & 0 & 1 \\
\hline Recurrence in Area C (+) $(n=1)$ & 0 & 1 & 0 & 0 \\
\hline Recurrence in Area D (+) $(n=3)$ & 0 & 0 & 3 & 0 \\
\hline
\end{tabular}

surface of the pancreatic neck; posterior, the pancreaticoduodenal coalescence fascia; medial, mesenteric vessels; and lateral, the uncinate process. Although the mesopancreas contains nerves, lymphatic vessels, and small blood vessels and is surrounded by fatty tissue, it has still not been clearly defined ${ }^{19}$. In other words, the mesopancreas includes the retroportal lamina, the second portion of pancreatic nerve plexus, and the mesopancreatoduodenum, which consists of the extra pancreatic nerve plexus, fatty tissue, lymphatic nodes and networks, and small blood vessels. The third and fourth portions of the duodenum and proximal jejunum form a common mesentery and are included in the "mesopancreas". The mesopancreas roughly corresponds to the area composed of the SMA margin based on the NCCN guidelines and the tissue left of the SMA margin until the ligament of Treitz (Area D) $)^{5-8,19,20,24}$.

Subsequently, we defined the MUP as the total extent of (1) the first portion of the pancreatic head nerve plexus (Area A), (2) the PV groove margin (Area B), (3) the SMA margin (Area C), and (4) left of the SMA margin to the ligament of Treitz (Area D) (Fig. 1b, 2). We then divided the MUP into 4 sections and stained them with TMDs of different colors. By doing so, the MUP could be subclassified. In fact, clear visualization of the surgical margin with various colors allows detailed delineation of a positive site on the MUP in individual patients, improved accuracy in diagnosis, and fewer surgical problems. As mentioned earlier, evaluating the surgical margin in conformational and nonserous organs is difficult. Evaluation of macroscopic and microscopic findings of the surgical margin does not always yield consistent results. In addition to the anatomical complexity of the resected sample, the loss of orientation of the divided sections of the MUP during paraffin-embedding often leads to great difficulty in evaluating the extremely important MUP. To resolve these issues, staining the resected samples with TMDs is useful. Staining with TMDs helps the surgical margin to be accurately diagnosed ${ }^{26,27}$.

The TMDs were originally developed for staining prostate and mammary glands to determine the outermost layer of these organs without a serosa ${ }^{26-28}$. The greatest benefits of TMDs are that they do not lose color during the staining process and that they retain their original color after staining. As a result, the true surgical margin can be accurately confirmed ${ }^{26-29}$. A variety of TMDs are 
available commercially ${ }^{28-30}$. Among the available stains, we adopted TMDs of the Davidson Marking System (Bradley Products, Inc.) because they stably adhere to the organ during histological staining and because the decolorization process is not complicated.

According to evaluation of the MUP by subclassification, the most common cancer-positive site on the surgical margin in $\mathrm{MUP}(+)$ cases was Area B (Table 1). The main reason for this result is that the PV groove margin is closest to the tumor in carcinomas of the pancreatic head. Another reason may be that fewer PDs with PV resection are performed at our institution than at other institutions $^{31-35}$. Thus, more aggressive adoption of PD with PV resection is highly recommended.

Although cancer cells can easily invade the PL $(n=19)$, only 2 cases $(4.4 \%)$ were Area $\mathrm{C}(+)$ in the present study (Table 1). This low rate may support circumferential PLsma-preserving PD in order to carry out R0 operations in selected patients, and promote better postoperative quality of life $\mathrm{f}^{36,37}$. Furthermore, TMD staining was able to be used to verify our preoperative multidetector CT diagnosis of obvious PLsma invasion.

For unknown reasons, no cases of the present study were in Area $\mathrm{A}(+)$.

In addition, Area $\mathrm{D}$ is the farthest from the pancreatic head. Therefore, Area D(+) may increase the likelihood of retroperitoneal dissemination. In support of this hypothesis, all initial sites of recurrence in Area $\mathrm{D}(+)$ in this study were localized, although early diagnosis of localized recurrence is difficult with CT (Table 2). In contrast, initial sites of recurrence in most $\mathrm{MUP}(+)$ cases other than Area $\mathrm{D}(+)$ cases were not localized. Contrary to our expectations ${ }^{38}$, no difference in disease-free survival time between cases with different $\mathrm{MUP}(+)$ sites was observed. This lack of difference may be due to the small number of patients in each subclass and the difficulty of using CT examination to distinguish recurrence from small postoperative scars. Hence, new methods of examination are needed for accurate and early judgment. Because Area D (+) represents an extension beyond the limit of the local disease, it may indicate the need for early aggressive adjuvant chemotherapy.

In similar studies reported earlier ${ }^{5,8-10,20,24}$, the rate of R1 operation was $23 \%$ to $63.0 \%$ and higher than in the present study. However, positive margin rate in sites similar to the MUP in patients undergoing R1 operation was $86.2 \%$ to $91.7 \%$, similar to the rate in the present study (90\%). In other words, in patients with carcinoma of the pancreatic head, the possibility that cancer cells remain in the MUP is high and makes the MUP the most important site for R0 operations. A similar tendency in the pattern of postoperative recurrence was indicated in other studies.

In 2 reports on the subclassification of sites similar to the MUP ${ }^{5,24}$, sites similar to Area C or Area D or both showed a high frequency of a positive margin $(57.6 \%$ and $53.1 \%$ ). This rate of a high cancer-positive margin was likely caused by insufficient dissection around the left side of SMA. Consequently, our left posterior approach for PD can make a decisive difference in the MUP (+) rate compared with conventional $\mathrm{PD}^{5-7,18}$.

Furthermore, 2 patients in the present study had multiple $\mathrm{MUP}(+)$ sites $(22.2 \%)$, mainly in Area B, at a rate lower than that described in other reports ${ }^{9,20}$. This result was due to the exclusion of patients with borderline resectable pancreatic cancer ${ }^{15}$ and patients with PLsma invasive pancreatic cancer and the execution of en bloc dissection via the left posterior approach for $\mathrm{PD}^{36,38}$.

\section{Conclusion}

By subclassifying the MUP with TMDs of different colors, we were able to confirm the regional characteristics of MUP(+). As a result, circumferential PLsma-preserving PD was able to be performed in R0 operations in selected patients while a better postoperative quality of life was maintained. Furthermore, Area $\mathrm{D}(+)$ represents an extension beyond the limit of the local disease and may indicate the need for early aggressive adjuvant chemotherapy.

Conflict of Interest: The authors have no financial conflicts of interest to disclose concerning this article.

\section{References}

1. Doi $\mathrm{R}$, Imamura $\mathrm{M}$, Hosotani $\mathrm{R}$, Imaizumi $\mathrm{T}$, Hatori $\mathrm{T}$, Takasaki K, Funakoshi A, Wakasugi H, Asano T, Hishinuma S, Ogata Y, Sunamura M, Yamaguchi K, Tanaka M, Takao S, Aikou T, Hirata K, Maguchi H, Aiura K, Aoki T, Kakita A, Sasaki M, Ozaki M, Matsusue S, Higashide S, Noda H, Ikeda S, Maetani S, Yoshida S: Surgery versus radiochemotherapy for resectable locally invasive pancreatic cancer: final results of a randomized multi-institutional trial. Surg Today 2008; 38: 1021-1028.

2. Imamura $M$, Doi $R$, Imaizumi $T$, Funakoshi $A$, Wakasugi H, Sunamura M, Ogata Y, Hishinuma S, Asano T, Aikou T, Hosotani R, Maetani S: A randomized multicenter trial comparing resection and radiochemotherapy for resectable locally invasive pancreatic cancer. Surgery 2004; 136: 1003-1011.

3. Sener SF, Fremgen A, Menck HR, Winchester DP: Pancreatic cancer: a report of treatment and survival trends for 100,313 patients diagnosed from 1985-1995, using the National Cancer Database. J Am Coll Surg 1999; 189: 1-7. 
4. Kato K, Yamada S, Sugimoto H, Kanazumi N, Nomoto S, Takeda S, Kodera Y, Morita S, Nakao A: Prognostic factors for survival after extended pancreatectomy for pancreatic head cancer: influence of resection margin status on survival. Pancreas 2009; 38: 605-612.

5. Gaedcke J, Gunawan B, Grade M, Szöke R, Liersch T, Becker H, Ghadimi BM: The mesopancreas is the primary site for R1 resection in pancreatic head cancer: relevance for clinical trials. Langenbecks Arch Surg 2010; 395: 451458.

6. Adham M, Singhirunnusorn J: Surgical technique and results of total mesopancreas excision (TMpE) in pancreatic tumors. Eur J Surg Oncol 2012; 38: 340-345.

7. Kawabata Y, Tanaka T, Nishi T, Monma H, Yano S, Tajima Y: Appraisal of a total meso-pancreatoduodenum excision with pancreaticoduodenectomy for pancreatic head carcinoma. Eur J Surg Oncol 2012; 38: 574-579.

8. Gockel I, Domeyer M, Wolloscheck T, Konerding MA, Junginger T: Resection of the mesopancreas (RMP): a new surgical classification of a known anatomical space. World J Surg Oncol 2007; 5: 44.

9. Rau BM, Moritz K, Schuschan S, Alsfasser G, Prall F, Klar E: R1 resection in pancreatic cancer has significant impact on long-term outcome in standardized pathology modified for routine use. Surgery 2012; 152: S103-111.

10. Konstantinidis IT, Warshaw AL, Allen JN, Blaszkowsky LS, Castillo CF, Deshpande V, Hong TS, Kwak EL, Lauwers GY, Ryan DP, Wargo JA, Lillemoe KD, Ferrone CR: Pancreatic ductal adenocarcinoma: is there a survival difference for R1 resections versus locally advanced unresectable tumors? What is a "true" R0 resection? Ann Surg 2013; 257: 731-736.

11. Mochizuki K, Gabata T, Kozaka K, Hattori Y, Zen Y, Kitagawa $\mathrm{H}$, Kayahara M, Ohta T, Matsui O: MDCT findings of extrapancreatic nerve plexus invasion by pancreas head carcinoma: correlation with en bloc pathological specimens and diagnostic accuracy. Eur Radiol 2010; 20: 1757-1767.

12. Tian $H$, Mori $H$, Matsumoto $S$, Yamada $Y$, Kiyosue $H$, Ohta M, Kitano S: Extrapancreatic neural plexus invasion by carcinomas of the pancreatic head region: evaluation using thin-section helical CT. Radiat Med 2007; 25: 141147.

13. Shioyama $Y$, Kimura $M$, Horihata $K$, Masuda M, Hagihira T, Okumura T, Yoshimi F, Amemiya R, Kishi K, Terada M, Sato M: Peripancreatic arteries in thin-section multislice helical CT. Abdom Imaging 2001; 26: 234-242.

14. Nakao A, Harada A, Nonami T, Kaneko T, Takagi H: Clinical significance of carcinoma invasion of the extrapancreatic nerve plexus in pancreatic cancer. Pancreas 1996; 12: 357-361.

15. Lupaşcu C, Andronic D, Grigorean VT, Ursulescu C: Mesopancreas first dissection during pancreaticoduodenal resection: selective approach or paradigm? Hepatogastroenterology 2014; 61: 463-468.

16. Nagakawa $Y$, Hosokawa $Y$, Osakabe H, Sahara $Y$, Takishita C, Nakajima T, Hijikata Y, Kasahara K, Kazuhiko K, Saito K, Tsuchida A: Pancreaticoduodenectomy with right-oblique posterior dissection of superior mesenteric nerve plexus is logical procedure for pancreatic cancer with extrapancreatic nerve plexus invasion. Hepatogastroenterology 2014; 61: 2371-2376.

17. Japan Pancreas Society: Classification of Pancreatic Carcinoma, 3rd English ed. 2011; Kanehara, Tokyo, Japan.

18. National Comprehensive Cancer Network: National Comprehenive Cancer Network Clinical Practice Guidelines in
Oncology (NCCN Guidelines ${ }^{\circledR}$ ). http://www.nccn.org/pr ofessionals/physician_gls/f_guidelines.asp. Accessed July 12,2016

19. Popescu I, Dumitrascu T: Total meso-pancreas excision: key point of resection in pancreatic head adenocarcinoma. Hepatogastroenterology 2011; 58: 202-207.

20. Delpero JR, Bachellier P, Regenet N, Le Treut YP, Paye F, Carrere N, Sauvanet A, Autret A, Turrini O, MongesRanchin G, Boher JM: Pancreaticoduodenectomy for pancreatic ductal adenocarcinoma: a French multicenter prospective evaluation of resection margins in 150 evaluable specimens. HPB (Oxford) 2014; 16: 20-33.

21. Aimoto T, Mizutani S, Kawano Y, Matsushita A, Yamashita N, Suzuki H, Uchida E: Left posterior approach pancreaticoduodenectomy with total mesopancreas excision and circumferential lymphadenectomy around the superior mesenteric artery for pancreatic head carcinoma. J Nippon Med Sch 2013; 80: 438-445.

22. Kurosaki I, Minagawa M, Takano K, Takizawa K, Hatakeyama K: Left posterior approach to the superior mesenteric vascular pedicle in pancreaticoduodenectomy for cancer of the pancreatic head. JOP 2011; 6: 220-229.

23. Dumitrascu T, David L, Popescu I: Posterior versus standard approach in pancreatoduodenectomy: a case-match study. Langenbecks Arch Surg 2010; 395: 677-684.

24. Liu L, Katz MH, Lee SM, Fischer LK, Prakash L, Parker N, Wang H, Varadhachary GR, Wolff RA, Lee JE, Pisters PW, Maitra A, Fleming JB, Estrella J, Rashid A, Wang H: Superior mesenteric artery margin of posttherapy pancreaticoduodenectomy and prognosis in patients with pancreatic ductal adenocarcinoma. Am J Surg Pathol 2015; 39: 1395-1403.

25. Ducreux M, Cuhna AS, Caramella C, Hollebecque A, Burtin P, Goéré D, Seufferlein T, Haustermans K, Van Laethem JL, Conroy T, Arnold D: Cancer of pancreas: ESMO Clinical Practice Guidelines for diagnosis, treatment and follow-up. Ann Oncol 2015; 26 (suppl 5): 56-68.

26. Chan KW, Lui I, Chung WB: Marking planes of surgical excision on specimens with mixture of India ink and acetone. J Clin Pathol 1989; 42: 893.

27. Williams AS, Hache KD: Recognition and discrimination of tissue-marking dye color by surgical pathologists: recommendations to avoid errors in margin assessment. Am J Clin Pathol 2014; 142: 355-361.

28. Paterson DA, Davies JD: Marking planes of surgical excision on breast biopsy specimens: use of artists' pigments suspended in acetone. J Clin Pathol 1988; 41: 1013-1016.

29. Williams AS, Dakin Haché K: Variable fidelity of tissuemarking dyes in surgical pathology. Histopathology 2014; 64: 896-900.

30. Kosemehmetoglu K, Guner G, Ates D: Indian ink vs tissue marking dye: a quantitative comparison of two widely used macroscopical staining tool. Virchows Arch 2010; 457: 21-25.

31. Noto M, Miwa K, Kitagawa H, Kayahara M, Takamura $\mathrm{H}$, Shimizu K, Ohta T: Pancreas head carcinoma: frequency of invasion to soft tissue adherent to the superior mesenteric artery. Am J Surg Pathol 2005; 29: 1056-1061.

32. Kitagawa H, Ohta T, Makino I, Tani T, Tajima H, Nakagawara $\mathrm{H}$, Ohnishi I, Takamura $\mathrm{H}$, Kayahara M, Watanabe $\mathrm{H}$, Gabata $\mathrm{T}$, Matsui $\mathrm{O}$, Zen Y: Carcinomas of the ventral and dorsal pancreas exhibit different patterns of lymphatic spread. Front Biosci 2008; 13: 2728-2735.

33. Kitagawa H, Tajima H, Nakagawara H, Makino I, Miyashita T, Shoji M, Nakanuma S, Hayashi N, Takamura H, Ohta T, Ohtake H: En bloc vascular resection for the 
treatment of borderline resectable pancreatic head carcinoma. Mol Clin Oncol 2014; 2: 369-374.

34. Nakao A, Kanzaki A, Fujii T, Kodera Y, Yamada S, Sugimoto $\mathrm{H}$, Nomoto S, Nakamura S, Morita S, Takeda S: Correlation between radiographic classification and pathological grade of portal vein wall invasion in pancreatic head cancer. Ann Surg 2012; 255: 103-108.

35. Poon RT, Fan ST, Lo CM, Liu CL, Lam CM, Yuen WK, Yeung C, Wong J: Pancreaticoduodenectomy with en bloc portal vein resection for pancreatic carcinoma with suspected portal vein involvement. World J Surg 2004; 28: 602-608.

36. Nguyen TC, Sohn TA, Cameron JL, Lillemoe KD, Campbell KA, Coleman J, Sauter PK, Abrams RA, Hruban RH, Yeo CJ: Standard vs. radical pancreaticoduodenectomy for periampullary adenocarcinoma: a prospective, random- ized trial evaluating quality of life in pancreaticoduodenectomy survivors. J Gastrointest Surg 2003; 7: 1-9.

37. Schniewind B, Bestmann B, Henne-Bruns D, Faendrich F, Kremer B, Kuechler T: Quality of life after pancreaticoduodenectomy for ductal adenocarcinoma of the pancreatic head. Br J Surg 2006; 93: 1099-1107.

38. Suenaga $M$, Fujii $T$, Kanda M, Takami H, Okumura N, Inokawa Y, Kobayashi D, Tanaka C, Yamada S, Sugimoto H, Nomoto S, Fujiwara M, Kodera Y: Pattern of first recurrent lesions in pancreatic cancer: hepatic relapse is associated with dismal prognosis and portal vein invasion. Hepatogastroenterology 2014; 61: 1756-1761.

(Received, January 4, 2016)

(Accepted, December 22, 2016) 